\title{
Desarrollo de estrategias digitales integrales y efectivas utilizando el viaje del visitante como framework clave
}

\section{Development of comprehensive and effective digital strategies using the visitor's journey as a key framework}

\section{Susana Funes}

NETSU- Estrategias Digitales para la Cultura. UAB. Email: $\underline{\text { susana@,funescentral.com }}$

\begin{abstract}
Resumen
Debido a la crisis sanitaria por la COVID-19 y el consecuente cierre de puertas, los museos enfocaron sus energías en los canales digitales para seguir en contacto con sus públicos. No obstante, gran parte de estas acciones no respondían a una estrategia digital como tal, sino a iniciativas puntuales y reactivas, quedando todavía pendiente un verdadero proceso de transformación digital. En el presente documento analizamos cómo abordar el desarrollo de una estrategia digital efectiva, concibiéndola de manera transversal y alineada con los objetivos, valores y actividad real de la organización, y orientándola a conectar de manera diferenciada con los distintos públicos y comunidades del museo. Para ello proponemos el enfoque del visitor journey como marco de trabajo e hilo conductor de la estrategia, con el fin de integrar todas las posibilidades que ofrecen las tecnologías digitales para el funcionamiento global del museo y la mejor relación con el usuario antes, durante y después de su visita. Este enfoque facilitaría priorizar acciones y vertebrar coherentemente la estrategia digital de un museo centrado en el usuario, al permitir, en primer lugar, obtener una visión holística y compartida de las expectativas y necesidades de los usuarios en cada fase; $y$, en segundo lugar, al facilitar el análisis transversal de todas las posibles estancias, procesos y actividades donde las tecnologías digitales podrían impulsar los objetivos del museo, en función de sus públicos y stakeholders.
\end{abstract}

Palabras clave: estrategia, digitalización, públicos, audiencias, visitorjourney

\footnotetext{
Abstract

Due to the COVID-19 global crisis and consequent lockdown restriction policies, museums focused their energies on digital channels to keep in touch with their audiences. However, most of these actions did not respond to a
} 
digital strategy as such, but to reactive, individual initiatives, leaving the true digital transformation process still pending. In this document we analyse the approach to developing an effective digital strategy, conceived in a transversal way and aligned with the objectives, values, and actual roles of the organization, and focused in connecting uniquely with the museums various audiences and communities. Therefore, we are proposing the visitor's journey approach as a framework and common thread for this strategy, in order to integrate the potential digital technologies have to offer to the museum's operation and its relationship with the user before, during and after his visit. This approach would facilitate prioritizing actions and coherently structuring the digital strategy of a user-centred museum, firstly by allowing to obtain a holistic and shared vision of the user's expectations and needs at each stage; and, secondly, by facilitating a cross-sectional analysis of all possible conditions, processes and activities where digital technologies could drive the museum's objectives, based on its audiences and stakeholders.

Keywords: strategy, digitization, audiences, user-centred, visitor-journey 


\section{Introducción}

La crisis sanitaria provocada por la COVID-19 y el consecuente cierre de museos e instituciones generó un inusitado impulso de los canales digitales, llegando incluso a pensarse que la transformación digital finalmente estaba alcanzando a la cultura. Como en otros sectores, los museos vieron en la web y las redes sociales una alternativa para seguir en contacto con sus públicos y aportar a la comunidad, compartiendo contenidos para sobrellevar el confinamiento.

Así, según un estudio realizado por The Network of European Museum Organisations (NEMO Office, 2021), entre 600 museos de 48 países, el 93\% de los consultados afirmó haber aumentado o iniciado servicios en línea durante la pandemia. De acuerdo con el informe, más del 75\% de los museos incrementó sus actividades en redes sociales o inició nuevas. Específicamente en España, según el sondeo de la Fundación Contemporánea (2021) “La Cultura en España 2020", el 74,7\% de las instituciones consultadas -no exclusivamente museos- adaptó actividades presenciales al formato digital, mientras que el $63,9 \%$ creó de manera extraordinaria nuevos contenidos y actividades digitales.

No obstante, la mayoría de estas incursiones no respondían a una estrategia digital como tal. De hecho, según el ya citado estudio de NEMO, casi el $40 \%$ de los museos indicó que no realizaba siquiera algún seguimiento o medición de sus actividades online. 8 de cada 10 museos informaron requerían apoyo adicional, más del $40 \%$ de ellos para el desarrollo de una estrategia digital. Y en el último sondeo realizado por Axiell/MuseWeb (2020), un 58\% de los encuestados describió las estrategias digitales de su institución como "aún en desarrollo" o "aún por venir".

Así podemos notar que el repunte digital durante la pandemia constó mayormente de iniciativas puntuales y reactivas, centradas especialmente en las redes sociales, que si bien sirvieron para mantener cierta actividad de cara al público, no tuvieron mayor impacto en el funcionamiento regular de las instituciones. Por el contrario, en muchos casos pusieron de manifiesto deficiencias estructurales que desde hace tiempo han afectado a los museos y, sobre todo, dejaron ver una falta de visión estratégica e integral de lo que las nuevas tecnologías pueden aportar a toda la organización.

En este sentido, aunque resulta muy meritoria la agilidad y la capacidad de adaptación que demostraron gran parte de los museos, llegó el momento de canalizar y capitalizar todo ese esfuerzo en un proceso de transformación digital más profundo, que ayude a garantizar la viabilidad y sostenibilidad de las organizaciones. A continuación, analizaremos cómo desarrollar una estrategia digital que no sólo aúne y dé coherencia a las iniciativas digitales nacidas a partir de la pandemia, sino sobre todo que apuntale integralmente la misión y objetivos de la organización. Para ello utilizaremos el enfoque del visitor journey como marco de trabajo e hilo conductor de la estrategia, con el fin de integrar todas las posibilidades que ofrecen las tecnologías digitales para el funcionamiento global del museo y la mejor relación con sus usuarios antes, durante y después de su visita. 


\section{Objetivos}

- Entender las bases y los principios básicos que debe cumplir una estrategia digital de un museo centrado en el usuario, para impulsar sus objetivos y apuntalar la relación con sus públicos y stakeholders.

- Comprender el concepto y las características esenciales del customer journey y su aplicación en los museos bajo el enfoque del visitor journey.

- Delinear los principios básicos para desarrollar una estrategia digital con el visitor journey como framework principal.

\section{Desarrollo de la innovación}

\subsection{El museo centrado en el usuario}

El concepto y visión del museo ha ido cambiando y adaptándose a los tiempos, pasando de ser una institución especialmente orientada a los objetos o bienes culturales -su conservación, custodia, investigación y difusión-, a enfocarse cada vez más en las personas (Samis y Michaelson, 2017). En el propio seno del International Council of Museum (ICOM) desde hace varios años se viene impulsando un cambio en la definición de museo que, si bien no se ha concretado en una formulación definitiva -el debate se ha extendido-, en su propuesta preliminar ya destacan conceptos como inclusión, participación y diálogo, caracterizando a los museos como "espacios democratizadores, inclusivos y polifónicos", "participativos y transparentes", que "trabajan en colaboración activa con y para diversas comunidades" (ICOM, 2019).

Respondiendo a tales exigencias y garantizar así su sostenibilidad y trascendencia como un activo valioso para la sociedad actual, los museos que ya estaban evolucionado antes de la crisis por la COVID-19 se han visto forzados a profundizar este vuelco. El objetivo es no sólo mantener su vigencia y relevancia ante sus públicos tradicionales, sino también atraer e implicar nuevas audiencias y especialmente interrelacionarse más estrechamente con sus diferentes stakeholders, en particular con las comunidades locales que, tras la caída del turismo, se convirtieron en foco de atención (L’Observatori dels Públics del Patrimoni Cultural de Catalunya, 2021) y, en ciertos casos, hasta en el centro de iniciativas para el bienestar y la superación postraumática.

Más allá de las medidas específicas para encarar la pandemia, el cambio de paradigma hacia el museo user-centred se espera prevalecerá, ya que de ello dependerá su vigencia y sostenibilidad. Las instituciones museísticas están pasando de orbitar alrededor del objeto cultural, sus espacios y patrimonio, a enfocarse en las personas: visitantes, públicos, usuarios, comunidades, etc.; es decir, los destinatarios finales de sus contenidos y actividades, quienes en última instancia dan sentido y trascendencia a su labor. El enfoque centrado en el usuario exige que la institución se vuelva más abierta, inclusiva y horizontal, favoreciendo a la colaboración y la cocreación. 
Este cambio o evolución conduce a su vez a un museo orientado a la experiencia. Un museo centrado en el usuario se convierte necesariamente en custodio y garante, ya no del objeto cultural, sino de las propias experiencias de sus usuarios. Es también en este sentido que los museos están pasando de ser "instituciones principalmente impulsadas por curadores" a convertirse en entidades enfocadas en las "necesidades de las audiencias y sus aprendizajes" (Kelly, 2004: 47-48). Así, las labores de mediación han ido afinándose, pasando de concebir a los museos como lugares de educación a lugares de aprendizaje, o más precisamente, entornos de aprendizaje de libre elección; es decir, un aprendizaje autodirigido, voluntario y guiado por las necesidades e intereses individuales (Kelly, 2004: 48), así como del contexto, antecedentes, conocimientos y experiencias previas del usuario.

Así, las instituciones user-centred buscan generar experiencias atractivas, valiosas y significativas, que conecten a los públicos con los objetos culturales. Para ello se procura ir más allá de lo meramente contemplativo, generando experiencias participativas y sociales, idealmente adaptadas al perfil e intereses del usuario. En ese sentido, ser user-centred requiere conocer en profundidad a públicos y audiencias digitales, para poder generar esas experiencias que conecten al usuario con el objeto cultural, tanto presencial como digitalmente. Para ello, a los tradicionales estudios de públicos se deberán sumar herramientas y técnicas mixtas de investigación, -analítica web, test de usuarios, eye tracking, encuestas online, analítica social, social listening, etc-. Y es en este marco que el enfoque del visitor journey puede ser una herramienta estratégica para contextualizar, asimilar coherentemente los datos y, sobre todo, para comprender las dinámicas de interacción fluida con el museo, por momentos como público, por momentos como participante, a veces audiencia, usuario, visitante o seguidor.

\subsection{Bases de una estrategia digital efectiva para el museo centrado en el usuario}

Históricamente las tecnologías digitales y sus ecosistemas asociados se han desarrollado en paralelo a nuevas formas de organización y vinculación muy alineadas con la idea del museo centrado en las personas: de lo unidireccional y asimétrico a lo multidireccional; de la verticalidad y rigidez jerárquica a la horizontalidad y fluidez de las redes; de lo lineal al rizoma; de la competencia a la cooperación (McAfee, 2009). En ese sentido, la transformación digital de los museos implica de alguna manera la asimilación de ciertos valores que viene impulsando la propia cultura digital, como la cooperación, la cocreación, la democratización de la información y el quiebre de jerarquías, para colocar a las personas en el centro de toda estrategia y acción, tal y como se ve también desde el user-centred design.

Bajo esta óptica, las nuevas tecnologías se convierten en un gran aliado para el museo usercentred. En el actual mundo hiperconectado -con 93\% de la población de Europa en línea (We Are Social/ Hootsuite, 2021)-, las tecnologías digitales presentan beneficios clave. En primer lugar, si se aprovechan el big data y las nuevas herramientas y técnicas de investigación y análisis de datos es posible conocer con muchísima más profundidad al usuario/visitante y su interacción fluida con el museo, digital y presencial. Asimismo, las técnicas y opciones digitales de microsegmentación y personalización ponen a disposición 
mecanismos más eficaces para alcanzar, no sólo a los públicos tradicionales de las instituciones culturales, sino especialmente a las anheladas nuevas audiencias, interactuando en su entorno natural como la red y el móvil, en constante interacción y diálogo entre lo real y lo virtual.

Con el conocimiento obtenido y aprovechando también los nuevos formatos y narrativas digitales -con todo su potencial visual y lúdico- es posible generar acciones y contenidos relevantes, adaptados a los perfiles, hábitos, acciones, expectativas e intereses del usuario, para generar esas experiencias significativas y memorables que lo vinculen con el objeto cultural y la institución. Asimismo, las nuevas tecnologías permiten impulsar y dar mayor visibilidad al trabajo científico tras bastidores, así como fortalecer la gestión interna, al contar con más datos para la toma de decisiones y mejores mecanismos de transparencia. Las nuevas tecnologías pueden incluso ayudar a generar nuevas fuentes de ingreso, no solo al ampliar el público visitante, o a través de la -aún en ciernes- monetización de contenidos, sino al dar paso a nuevas formas de apoyo como el crowdfunding y la activación de nuevas generaciones de patronos.

Con todo este potencial y opciones, la transformación digital requiere contar con una visión estratégica y desarrollar las capacidades internas que permitan integrar transversalmente los canales digitales y no digitales, para impulsar los objetivos de la organización. La digitalización no debe guiarse por modas o tendencias. No implica que todo museo tenga que estar necesariamente en cada nueva red social, ni abrazar per se cualquier nueva tecnología. Una estrategia digital efectiva es aquella que se concibe alineando cada una de las iniciativas y acciones a realizar en función de la misión, los objetivos organizacionales y los stakeholders asociados a esos objetivos.

Para ello es necesario centrarse en las tecnologías que puedan impulsar tres aspectos principales: primero, fortalecer y optimizar las capacidades y procesos internos, apuntalando las áreas clave de la organización -la razón de ser-; segundo, estrechar y fortalecer la relación con los diferentes públicos, así como optimizar la experiencia de visitantes / usuarios; y, tercero, mejorar la relación de la organización con su entorno, comunidades locales y la sociedad en general. Este último aspecto ha cobrado peso en los últimos años, llegando a su punto más alto tras la pandemia, cuando se ha hecho patente el rol social de los museos, su relación con las comunidades locales y su posible impacto para el bienestar y recuperación postraumática de los ciudadanos.

El museo centrado en las personas requiere desarrollar una estrategia digital integral, alineada con los objetivos, valores y actividad real de la organización, pero especialmente diseñada para conectar de manera diferenciada con los distintos públicos, comunidades y stakeholders. No hay fórmulas mágicas ni únicas. Cada organización deberá analizar su propuesta de valor y las características de sus públicos y audiencias, para evaluar cómo agregar una capa digital que catapulte la actividad natural de la organización: desde la atención al público y la experiencia del visitante, a la gestión y toma de decisiones, pasando por la investigación, curación, catalogación, etc. 


\subsection{El viaje del visitante o visitor-journey como framework estratégico}

En la actual sociedad conectada no tiene sentido hacer una diferenciación estanca entre el visitante físico y el usuario web, entre los públicos presenciales y las audiencias digitales, ya que las formas de relación con el museo son hoy fluidas, alternándose los contactos on y off. Un visitante pudo haber comenzado su interacción con el museo en línea como un usuario web, para posteriormente ir de forma física a una exposición, desde allí interactuar en redes sociales y, una vez terminada la visita, seguir vinculado digitalmente con la institución. En ese sentido, una estrategia digital exitosa deberá ser integral, cruzando tranversalmente toda la organización según los objetivos, los valores y la actividad de la institución, a la vez que omnicanal y centrada en el usuario; esto es, optimizada según sus necesidades y expectativas a lo largo de todos los canales y puntos de contacto digitales y físicos.

Dentro de este marco y con el fin de definir esa estrategia digital efectiva, el enfoque del customer journey -originalmente utilizado en el desarrollo de productos y muy extendido en la experiencia de usuario (UX) y el user-centred design- puede ser de gran utilidad para analizar transversalmente todas las posibles estancias, procesos o actividades donde las tecnologías digitales podrían impulsar los objetivos del museo, en función de los públicos y stakeholders con los que se relaciona para cumplir su misión.

Este enfoque facilitaría priorizar acciones y vertebrar coherentemente la estrategia digital a partir del análisis, en primera instancia, del viaje del visitante o visitor journey; es decir, visualizando cómo son las distintas interacciones del usuario/visitante con la institución y cuáles son sus requerimientos a lo largo de todo su recorrido. Para fines explicativos y por tratarse de la clave del museo centrado en el usuario, aquí nos enfocaremos en el visitor journey. No obstante, el modelo de viajes o journeys puede y debe adaptarse a cada uno de los stakeholders, pudiendo analizarse, según los objetivos prioritarios del museo, el viaje del empleado o colaborador interno, el viaje del patrono y de cada uno de los actores clave involucrados en el quehacer de la organización.

Tal y como lo define Nielsen Norman Group, uno de los referentes en el diseño centrado en el usuario, el journey map es, en términos muy simples, la "visualización del proceso que atraviesa una persona para lograr una meta" (Gibbons, 2018). Aplicándolo al museo, podemos definir el visitor journey map como la representación visual de un viaje arquetípico, la visualización del recorrido, los pasos y acciones que realiza un usuario tipo al interactuar con el museo por cualquiera de sus canales físicos o digitales. El mapa del viaje del visitante documentaría, por ejemplo, qué pasos sigue para saber de la existencia del museo, conocer su oferta, planificar su visita, comprar la entrada, ingresar al museo, moverse por las salas, conocer sobre las obras, ir al servicio, tomar un café, participar en alguna actividad, comprar una postal o souvenir, etc. De todas estas interacciones se busca identificar qué necesita a cada paso, qué resultados obtiene, qué desajustes existen en ese recurrido y qué mejoras se pueden introducir.

En su forma más básica, el diseño de este mapa comienza con la compilación sobre una línea de tiempo de los objetivos y acciones de un usuario tipo o $<<$ visitor persona $>>$. A estas 
acciones se le añaden pensamientos y emociones para crear una narrativa funcional, que nos permita ponernos en los zapatos del visitante. Es esta narrativa, ya condensada y pulida, la que permite visualizar el recorrido, para así comprender mejor las expectativas del usuario y poder mejorar su experiencia, identificando los llamados $<<$ momentos de verdad $>>$. Estos momentos pueden ser $<<$ wow moments $>>$, es decir, puntos de interacción que, siendo percibidos como relevantes, ofrecen una experiencia y emoción positiva y significativa para el usuario; o <<pain moments $>>$, puntos de fricción en acciones importantes para el usuario que ofrecen una experiencia insatisfactoria o no acorde con las expectativas. En suma, el mapa del viaje del visitante facilita comprender los pasos que sigue un usuario para conseguir un objetivo y cuál es su experiencia, pensamientos y emociones, así como el grado de satisfacción o frustración.

El gran aporte del enfoque del viaje del visitante en el diseño de estrategias digitales es, en primer lugar, que da coherencia y sistematicidad a cada acción o iniciativa que se realice en función del usuario/visitante. Ya no se piensa de manera individual en una app, una red social, un folleto o una newsletter, sino en un entramado fluido de canales y puntos de contacto on y off, así como en posibles interacciones. Ante ellas, el museo tendría que responder de manera integral e igual de fluida, a través de recursos físicos y digitales (omnicanal), generando experiencias consistentes y satisfactorias antes, durante y después de la visita, hasta reiniciar el ciclo con la recomendación o el regreso.

El visitor-journey facilita también comprender que el museo no se dirige a un público homogéneo o indiferenciado. Cada visitante/usuario es diferente, por lo que resulta vital delinear distintos recorridos según diversos visitor-personas. Una $<<$ persona $>>$ es una descripción ficticia, pero realista, de un usuario típico u objetivo del producto, del sistema (Haryley, 2015) o, en este caso, de la institución. La Persona se concibe como un arquetipo (Cooper, 1999), una imagen compuesta de personas reales que son usuarios o posibles usuarios del museo. Se trata de un avatar creado a partir de lo que se conoce del visitante a través de estudios de públicos, entrevistas directas, focus groups, así como del comportamiento observado en cualquier otra instancia. En este sentido conviene delinear diferentes journeys correspondientes a los distintos perfiles prioritarios del museo. Así, por ejemplo, un turista amante del arte tendrá un recorrido, unas necesidades y expectativas muy diferentes a una madre con dos pequeños, una pareja joven, un visitante primerizo o un profesor que prepara una visita escolar.

Aunque el visitor journey mapping debe realizarse específicamente para un contexto y con un objetivo específico -un museo, una visitor persona, un escenario- como esbozaremos en breve, para fines explicativos aquí presentamos un modelo genérico establecido para ilustrar cómo sería aplicar el enfoque del journey en el diseño de una estrategia digital y un plan de contenidos, que permita acompañar al usuario y apuntalar su viaje antes, durante y después de la visita (Ver figura 1: Modelo ilustrativo Visitor Journey).

Para ello, dibujamos las posibles interacciones que pudiera tener un visitante desde que comienza su búsqueda de opciones de salida y tiene el primer contacto con el museo 
(búsqueda de plan), pasando por los preparativos y arreglos para su visita (preparación), la propia llegada al museo y recorrido por salas y exposiciones (visita), hasta su retiro a casa, cuando aún encontrándose fuera del museo puede mantener el contacto con la institución (posvisita).

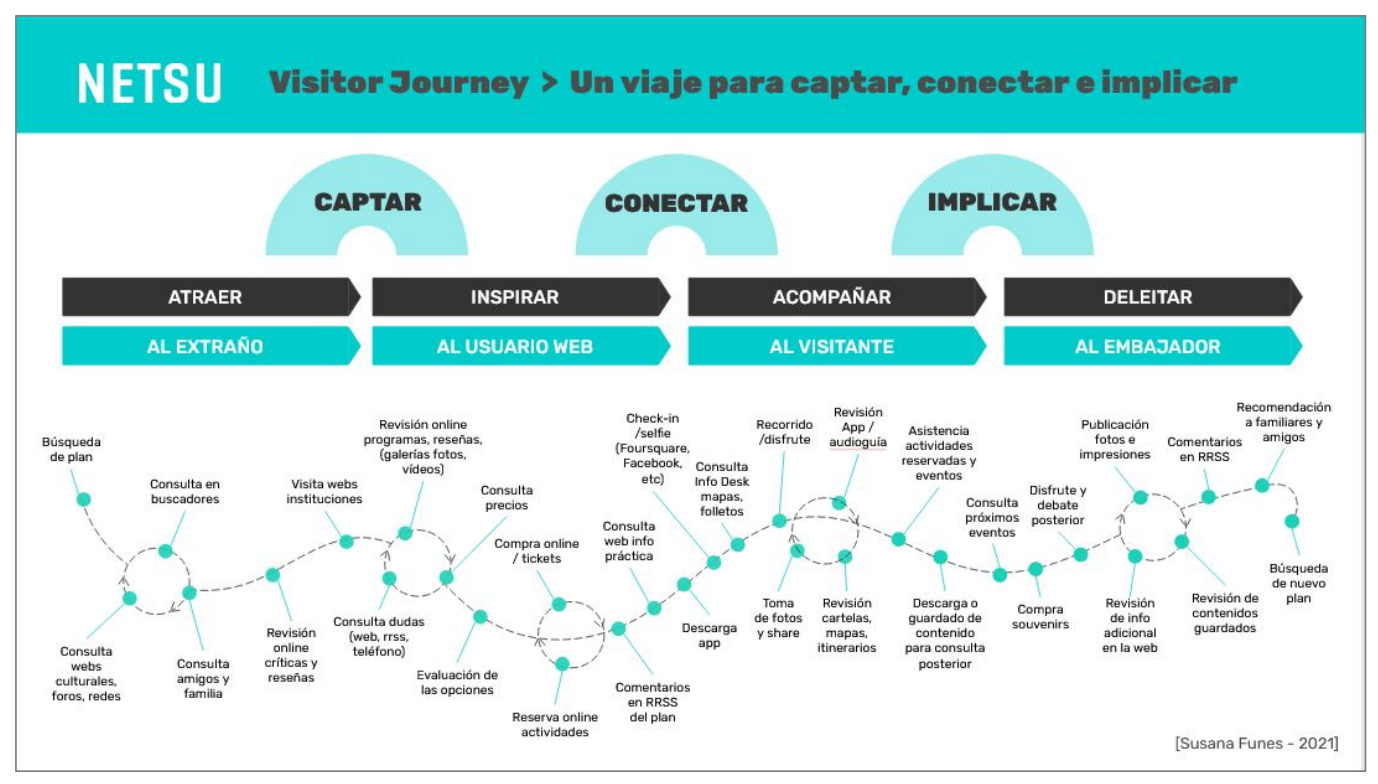

Fuente: Funes (2021)

Fig. 1 Modelo ilustrativo Visitor Journey

Cabe resaltar que el objetivo de este enfoque -y por tanto lo que se ilustra en el journey mapes poder captar, es decir alcanzar y atraer al usuario; conectar con él mediante contenidos relevantes y memorables; e implicarlo o vincularlo a la institución a través de experiencias valiosas y significativas. En ese proceso, la propia figura del usuario/visitante va evolucionando a medida que interactúa con el museo. La meta es establecer una estrategia digital y un plan de contenidos ajustado a las necesidades e interacciones del usuario, que permita captar al $<<$ extraño $>>$, a un potencial visitante que no conocía o que nunca había visitado el museo; inspirar al $<<$ usuario web $>>0$ al seguidor en redes sociales motivando la visita; acompañar al $<<$ visitante $>>$ en toda su exploración, mejorando la calidad de su experiencia; y deleitarlo tras su partida convirtiéndolo en $<<$ embajador $>>$. Como puede verse en la segunda ilustración (Ver figura 2: Modelo ilustrativo Visitor Journey Contenidos y canales), habiendo establecido puntos y momentos de interacción clave, así como las necesidades del usuario para cada momento, se puede establecer un plan de contenidos ajustado para cada fase y canal. 


\section{NETSU Visitor Journey > Contenidos y canales}

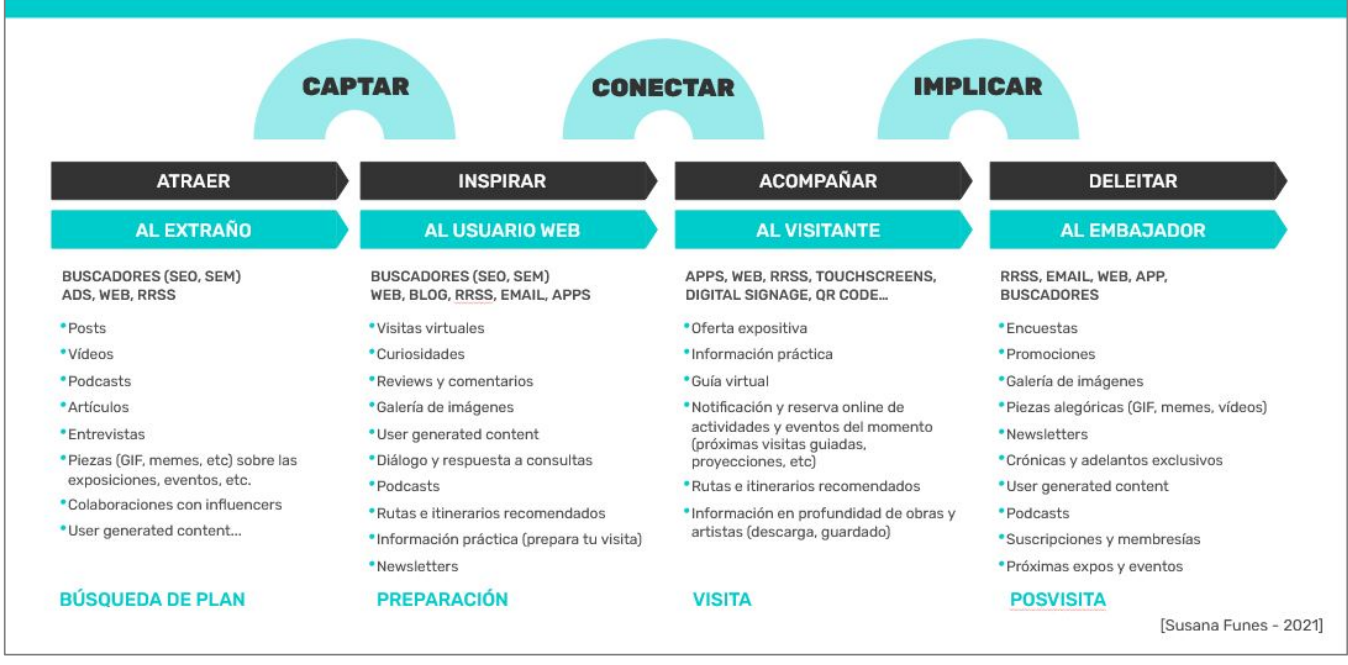

Fuente: Funes (2021)

Fig. 2 Modelo ilustrativo Visitor Journey - Contenidos y Canales

El mapeo completo del viaje del visitante permite tener una visión holística de la experiencia del usuario e identificar puntos clave y de alto valor donde crear contenidos, desarrollar recursos y servicios, según las necesidades, preocupaciones, comportamientos y expectativas específicas de los diferentes públicos y comunidades. Si se realiza correctamente, el journey map puede revelar también aspectos poco conocidos del visitante, así como puntos débiles, brechas o inconsistencias que resolver dentro del recorrido. En ese sentido, el visitor journey map es un excelente punto de partida para delinear una estrategia digital user-centred, al responder por dónde empezar y evidenciar las principales áreas de fricción. La narrativa y visualización ayuda, además, a transmitir la información de forma concisa, clara y memorable a lo largo de toda la organización, permitiendo desarrollar una visión compartida de la experiencia del usuario y facilitando alinear a los diferentes departamentos y actores para mejorarla.

\subsection{Aspectos básicos para aplicar el enfoque del visitor-journey}

Al ser un documento estratégico, el desarrollo de los visitor journeys suele incluir diversas sesiones de trabajo para la definición de objetivos, la recolección y el análisis de la información existente y la segmentación públicos, hasta llegar a la realización de workshops, con la participación de todos los departamentos de la organización. Si bien explicar detalladamente la metodología está fuera del alcance del presente documento -puede consultarse Devine (2015), Grohe y Mann (2019), Gibbons (2018) y especialmente Kalbach (2016)-, esbozamos algunos aspectos básicos.

Normalmente los mapas de viaje pueden estructurarse de diferentes maneras, según quién lo aplique, bajo qué contexto y para qué objetivo. No obstante, en general se pueden identificar al menos cinco elementos comunes (Ver Fig 3 - Customer-Journey Map Template -Nielsen Norman Group) 


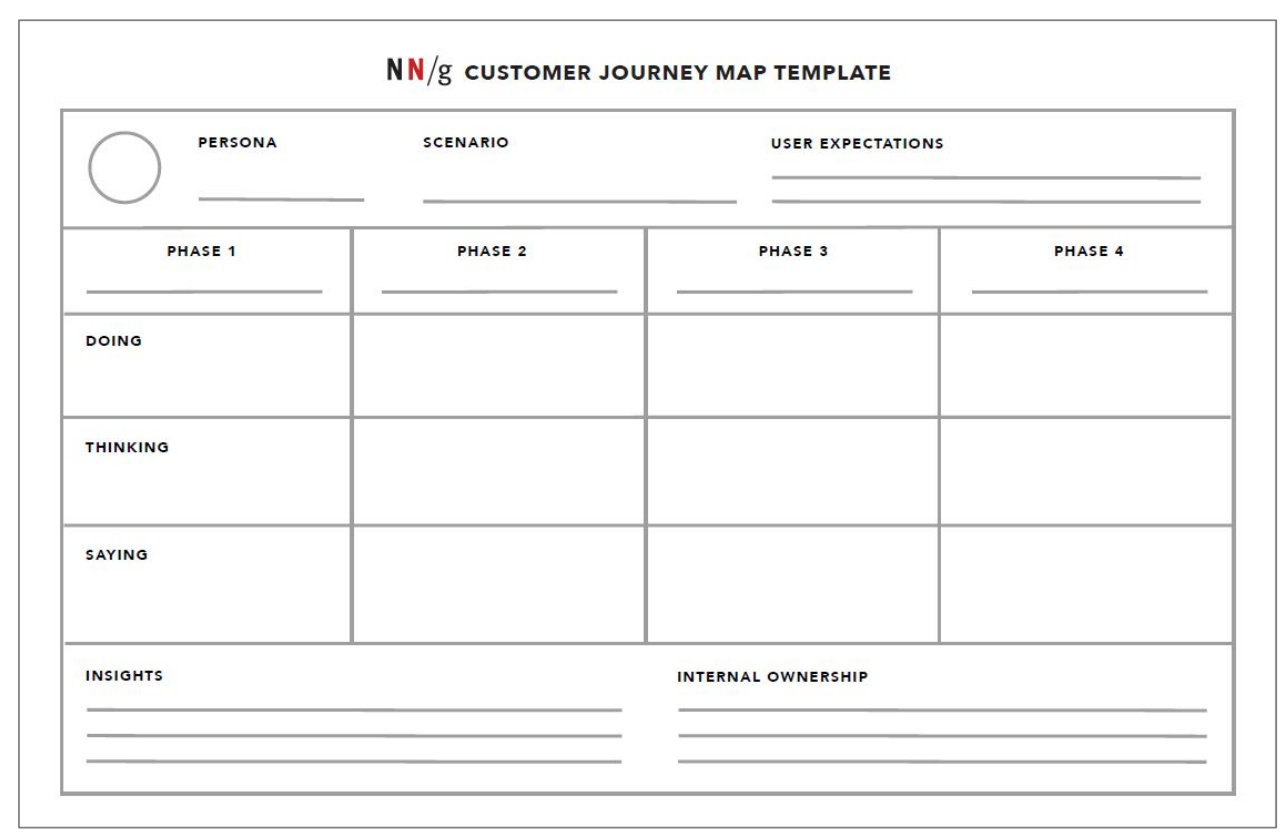

Fuente: Gibbons (2018)

Fig. 3 Customer-Journey Map Template - Nielsen Norman Group

Persona. Cada itinerario se diseña para un actor concreto, una visitor-persona (arquetipo) que es quien protagoniza o experimenta el recorrido. Es el punto de vista para analizar la experiencia de interacción con la institución. En general conviene alinear esta visitorpersona a los diferentes perfiles de usuario del museo, por ejemplo: una madre, un turista amante del arte, una pareja joven, etc.

Escenario. Es la experiencia específica a mapear que consistirá en una secuencia de eventos o acciones para lograr un objetivo. En el caso de un museo podría ser la visita general a la institución o una exposición, pero también puede ser un objetivo aún más específico, como la compra de una entrada.

Fases / Timeline. Son las diferentes etapas del trayecto o itinerario al más alto nivel, que permiten organizar en un sentido temporal el resto de la información: acciones, pensamientos y emociones. Las etapas pueden variar de un escenario a otro. No obstante, para el caso de los museos en términos generales pueden identificarse tres principales: previsita, visita y posvisita.

Acciones, pensamientos y emociones. El corazón del visitor journey y lo que articula toda la narrativa es lo que el usuario hace, piensa y siente en el recorrido. Para completar estos aspectos es vital basarse en conocimiento previo del visitante (investigaciones cualitativas, entrevistas, focus groups, estudios de público, observación contextual, shadowing, etc.). El grado de especificidad y profundidad dependerá del propósito del mapa.

Touchpoints y canales. Se deben alinear los puntos de contacto o interacción entre la Persona y cualquier agente o artefacto del museo, así como los canales o medios utilizados para hacerlo. Estas interacciones tienen lugar en un momento y contexto determinado, con la 
intención de satisfacer una necesidad específica. Además de los canales tradicionales del museo se deberán incluir los diferentes canales online: página web, redes sociales, aplicaciones móviles, email, kioskos interactivos, juegos online, etc. La meta es ofrecer una experiencia consistente y satisfactoria independientemente del canal donde comience, se desarrolle o finalice (omnicanal), por lo que el análisis de los puntos de contacto resulta crucial, ya que frecuentemente es aquí donde se descubren las mayores inconsistencias.

Insights y oportunidades. Son los conocimientos adquiridos a partir del mapeo acerca de cómo se puede optimizar la experiencia del usuario: brechas en la experiencia -muy comunes en los viajes onmnicanal- y cualquier información que surja del proceso. Un elemento importante a indicar es la $<<$ propiedad $>>$ de cada tarea o cambio que se desee implementar; es decir, explicitar quién es el responsable o quién está a cargo de qué aspecto del viaje del visitante.

Tanto la estructura, como la especificidad y profundidad de los visitor-journey dependerán de la institución y los objetivos planteados.

\section{Resultados: El visitor-journey map como herramienta pos-COVID-19}

Desde marzo de 2020 museos en todo el mundo debieron cerrar sus puertas debido al coronavirus y las consecuentes restricciones sanitarias para controlar la pandemia. Un año después, todavía muchas instituciones están en fases iniciales de reapertura y, en general, todos están enfrentándose al desafío de cómo volver a las actividades regulares con seguridad, cómo admitir nuevamente visitantes -y recuperarlos-, cómo dar continuidad a las iniciativas emprendidas durante la pandemia, cómo integrar lo aprendido y de qué manera profundizar en la digitalización y en ese objetivo de centrarse en el usuario, así como en el rol social del museo, reivindicado como centro de bienestar y recuperación postraumática.

Dentro de este marco, el enfoque del visitor-journey puede ser una herramienta estratégica para la toma de decisiones, al permitir valorar alternativas y canalizar esfuerzos, en función, precisamente, de las necesidades y expectativas del usuario. Como una suerte de "beneficio colateral" de la pandemia, la digitalización se ha acelerado en todos los sectores. Y el enfoque del visitor-jorney puede ayudar a guiar la transformación digital de los museos, permitiendo desarrollar una estrategia integral, consistente y orientada al usuario, que impulse los objetivos de la organización.

Además, el visitor-journey map puede ser la clave para adaptarse a la nueva normalidad, al permitir al museo analizar los diferentes recorridos y evaluar de forma sistemática los principales canales y puntos de contacto entre usuario y museo. Podría arrojar luz acerca de cómo sustituir puntos de contacto físico -taquillas, tickets, membresías, audioguías compartidas e, incluso, pantallas táctiles e interactivos-, en favor de pagos contactless, entradas y tarjetas de membresía digitales, así como otros recursos digitales accesibles por el usuario desde su propio dispositivo móvil, como se muestra en la siguiente ilustración. (Ver figura 4: Modelo ilustrativo Visitor Journey - Adaptándonos a la nueva normalidad) 


\section{NETSU Visitor Journey > Adaptándonos a la nueva normalidad}

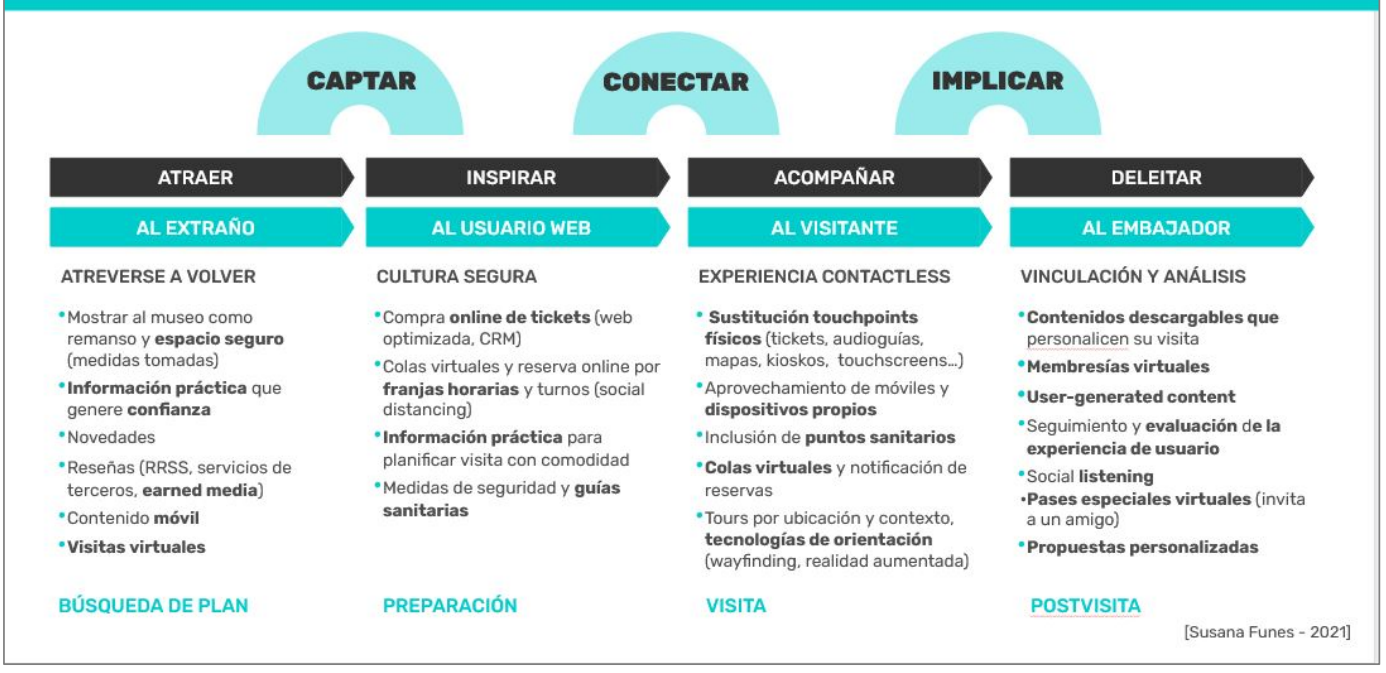

Fuente: Funes (2021)

Fig. 4 Modelo ilustrativo Visitor Journey-Adaptándonos a la nueva normalidad

También permitiría darle prioridad a ciertos procesos digitales que podrían ser cruciales para la supervivencia del museo, como reducir el número de pasos para completar una transacción en el sitio web, o implementar un software de reserva en línea, con horarios específicos de acceso, que ayude a cumplir los requerimientos de aforo y normas de seguridad. Con la visualización del visitor-journey se facilitaría la realización de ajustes en itinerarios y diferentes servicios, para garantizar así la seguridad y bienestar de visitantes, empleados y colaboradores.

\section{Conclusiones}

Desde hace varios años se viene dando un cambio en la concepción de museo, pasando de ser una institución centrada en el objeto cultural -su protección, custodia, conservación y difusión- a una entidad más centrada en las personas -públicos, usuarios y comunidades. Dentro de esta evolución, las tecnologías digitales ofrecen una serie de ventajas para ayudar a los museos a cumplir con sus objetivos organizaciones y, especialmente, para centrarse en el usuario, al permitir generar experiencias significativas que lo conecten con el objeto cultural.

Debido a la COVID-19 y las restricciones sanitarias, museos en todo el mundo debieron cerrar sus puertas, abocándose a los canales digitales para mantenerse activos y seguir en contacto con sus públicos. No obstante, en su gran mayoría, tales iniciativas no respondían a 
un proceso de transformación digital ni a una estrategia digital estructurada, sino a acciones puntuales y reactivas.

Superada la fase más crítica de la pandemia, llegó el momento de canalizar y capitalizar todos los esfuerzos. En ese sentido, la definición de una estrategia digital resulta vital no sólo para aunar y dar coherencia a las iniciativas nacidas durante la pandemia, sino sobre todo para emprender una verdadera digitalización. Un proceso de transformación digital depende del desarrollo de una estrategia integral, alineada con los objetivos, valores y actividad de la organización, pero especialmente diseñada para conectar de manera diferenciada con los distintos públicos y comunidades.

Para ello, el enfoque del visitor-journey se plantea como una herramienta clave al permitir conocer en mayor profundidad la experiencia del visitante, a lo largo de todas sus interacciones con el museo antes, durante y después de la visita. Este enfoque facilitaría priorizar acciones y vertebrar coherentemente la estrategia digital de un museo centrado en el usuario, al permitir, por un lado, obtener una visión holística y compartida de las expectativas y necesidades de los usuarios en cada fase; y, por otro, al facilitar el análisis transversal de todas las posibles estancias, procesos o actividades donde las tecnologías digitales podrían impulsar los objetivos del museo, en función de sus públicos y stakeholders.

Si bien el enfoque del visitor-journey se ha venido introduciendo en los museos con diferentes objetivos puntuales, ahora puede adaptarse como una herramienta estratégica en procesos de transformación digital. Su utilización permitiría comprender las dinámicas de interacción fluida del usuario con el museo -ora como público, ora como participante, audiencia, usuario, visitante o seguidor-, para así diseñar una estrategia digital efectiva, que potencie los objetivos y actividad real de la institución y permita centrarse en el usuario, ofreciendo una experiencia omnicanal y optimizada, según sus necesidades y expectativas, a lo largo de todos los canales y puntos de contacto del museo.

\section{Referencias}

Axiell/MuseWeb. (2020). "Report: Culture at a Crossroads, Digital Transformation in the Age of COVID-19" en Axiell, 9 de diciembre. <https://www.axiell.com/report/report-culture-at-acrossroads-digital-transformation-in-the-age-of-covid-19/> [Consulta: 15 de enero de 2021]

Buiss, J. (2016). "How the Smithsonian Built Their Journey Map, with Samir Bitar" en Customer Bliss, 6 de mayo. <https://www.customerbliss.com/smithsonian-built-journey-map-samir-bitar-cb4/> [Consulta: 28 de noviembre de 2020]

Chan, S. (2015). "Visitor journey mapping at ACMI" en ACMI Labs, 15 de diciembre. $<$ https://labs.acmi.net.au/visitor-journey-mapping-at-acmi-286e985bf4ae > [Consulta: 17 de enero de 2021]

CoOPer, A. (1999). The Inmates Are Running the Asylum. Indianapolis: Sams.

Devine, C. (2015). “The Museum Digital Experience: Considering the Visitor's Journey” en MWA2015: Museums and the Web Asia 2015, 15 de agosto. 
$<$ https://mwa2015.museumsandtheweb.com/paper/the-museum-digital-experience-considering-thevisitors-journey/> [Consulta: 30 de diciembre de 2020]

EUROPEAN COMMISION. (2017). Study on audience development. How to place audiences at the centre of cultural organisations: final report. Publications Office of the EU, 22 de marzo. $<$ https:/op.europa.eu/en/publication-detail/-/publication/cc36509d-19c6-11e7-808e01aa75ed71a1> [Consulta: 20 de enero de 2021]

FALK, J. Y DiERKING, L. (2016). The Museum Experience Revisited. New York: Routledge.

Fundación Contemporánea. (2021). La Cultura en España 2020. Observatorio de la cultura, enero 2021. $<$ https://www.lafabrica.com/wp-images/Informe-OC_2020.pdf > [Consulta: 19 de febrero de 2021]

FunES, S. (2019a). "Estrategias digitales para el arte y la cultura: ¿Cómo captar a las nuevas audiencias conectadas". The Valley Barcelona. En Seminario Cultura 4.0: Estrategias digitales para el arte y la cultura en un mundo hiperconectado. (Ponencia inédita).

FunES, S. (2019b). "Transformación digital del sector cultural, la clave para cautivar nuevos públicos" en The Valley Blog, 9 de julio. <https://thevalley.es/blog/transformacion-digital-sector-culturalclave-nuevos-publicos/> [Consulta: 20 de enero de 2021]

GibBons, S. (2018). "Journey Mapping 101" en Nielsen Norman Group, 9 diciembre. $<$ https://www.nngroup.com/articles/journey-mapping-101/> [Consulta: 14 de enero 2021]

Grohe, M. y MANN, L. (2017). "MCNPro - User Journey Mapping” en MCN Blog, 19 de septiembre. $<$ http://mcn.edu/mcnpro-user-journey-mapping/ > [Consulta: 10 de enero de 2021]

GroHe, M y MANN, L. (2019). "Walking in the shoes of our visitors: Human-centered design and organizational change at the Isabella Stewart Gardner Museum”, en MW19: $M W 2019,3$ de abril. $<$ https://mw19.mwconf.org/paper/walking-in-the-shoes-of-our-visitors-human-centered-designand-organizational-change-at-the-isabella-stewart-gardner-museum/> [Consulta: 2 de enero de 2021]

Harley, A. (2015). "Personas Make Users Memorable for Product Team Members" en Nielsen Norman Group, 16 de febrero. <https:/www.nngroup.com/articles/persona/> [Consulta: 14 de enero 2021]

ICOM. (2019). "ICOM announces the alternative museum definition that will be subject to a vote" en ICOM, 25 de julio. <https://icom.museum/en/news/icom-announces-the-alternative-museumdefinition-that-will-be-subject-to-a-vote/> [Consulta: 10 de febrero de 2021]

KalBach, J. (2016). Mapping Experiences: A Guide to Creating Value Through Journeys, Blueprints and Diagrams. Sebastopol, CA: O’Reilly Media.

KAPLAN, K. (2016). "When and How to Create Customer Journey Maps" en Nielsen Norman Group, 31 de julio. <https://www.nngroup.com/articles/customer-journey-mapping/> [Consulta: 14 de enero 2021]

Kelly, L. (2004). "Evaluation, Research and Communities of Practice: Program Evaluation in Museums" en Archival Science, 4, p. 45-69.

L'Observatori dels Publics del Patrimoni Cultural de Catalunya. (2021). Museus i equipaments patrimonials que van incrementar visitants l'agost de 2020 respecte 2019. Anàlisi de casos. Girona, enero de 2021. <https://interaccio.diba.cat/sites/interaccio.diba.cat/files/museusequipaments-patrimonials-augmentar-public.pdf $>$ [Consulta: 1 de febrero de 2021] 
MCAFEE, A. (2009). Enterprise 2.0: New Collaborative Tools for Your Organization's Toughest Challenges. Cambridge: Harvard Business Press.

Network of European Museum Organisations. (2021). Follow-up survey on the impact of the COVID-19 pandemic on museums in Europe - Final Report. <https://www.nemo.org/fileadmin/Dateien/public/NEMO_documents/NEMO_COVID19_FollowUpReport_11.1.2 021.pdf $>$ [Consulta: 11 de enero de 2021]

Samis P. y Michaelson, M. (2017). Creating the Visitor-Centered Museum. New York: Routledge.

Villaespesa, E. y AlvareZ, A. (2019). "Visitor journey mapping at the Museo Nacional ThyssenBornemisza: bringing cross-departmental collaboration to build a holistic and integrated visitor experience" en Museum Management and Curatorship, vol. 35, issue 2, p. 125-142. $<$ https://doi.org/10.1080/09647775.2019.1638821> [Consulta: 19 de diciembre de 2020]

Villeneuve, P. (2013). "Building museum sustainability through visitor-centered exhibition practices” en International Journal of the Inclusive Museum, vol. 5, issue 4, p. 37-50.

We Are Social y HoOtSuite. (2021). Digital 2021.<https://wearesocial.com/digital-2021> [Consulta: 22 de enero de 2021] 